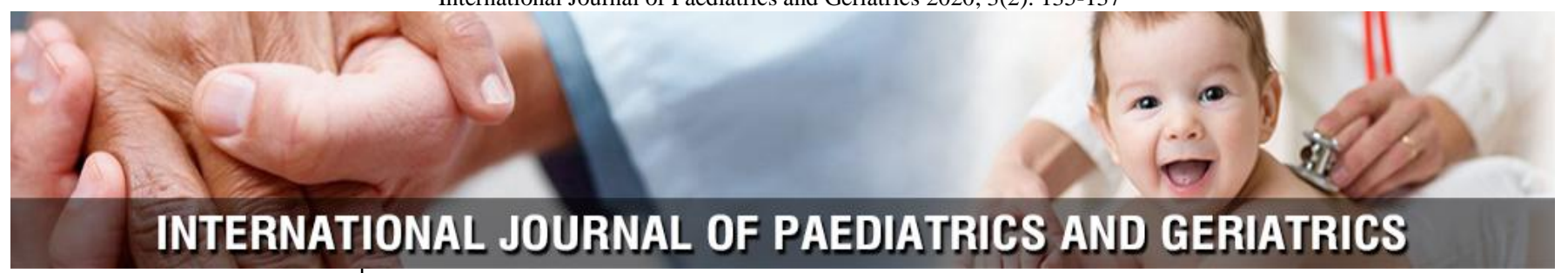

P-ISSN: 2664-3685

E-ISSN: 2664-3693

IJPG 2020; 3(2): 135-137

Received: 05-05-2020

Accepted: 08-06-2020

Dr. Divyarani DC

Associate Professor,

Department of Pediatrics,

Kodagu Institute of Medical

Sciences, Madikeri, Karnataka, India

Dr. Anupama Pandey Senior Resident, Department of Pediatrics, JSS Medical College, Mysore, Karnataka, India
Corresponding Author: Dr. Anupama Pandey Senior Resident, Department of Pediatrics, JSS Medical College, Mysore, Karnataka, India

\section{The predictors of MDR organisms causing sepsis in neonates born in a tertiary care hospital}

\author{
Dr. Divyarani DC and Dr. Anupama Pandey
}

DOI: https://doi.org/10.33545/26643685.2020.v3.i2c.105

\begin{abstract}
There have been various studies in the direction of changing trends of antimicrobial sensitivity of organisms causing neonatal sepsis, though there have been no cumulative studies to demonstrate the risk factors, clinical profiles of neonates suffering from MDR and non MDR sepsis. Also there is no comparative data on the outcomes of MDR and non MDR neonatal sepsis. The present study aims to fill the aforementioned gaps. A prospective hospital based study over a period of one year was carried out at neonatal intensive care unit of our hospital, a tertiary care center. At least $20 \%$ deliveries require nursery admission. All neonates born at the hospital and presenting with signs and symptoms of sepsis or born to mothers with potential risk factors for sepsis were investigated for sepsis. The most common presenting symptom in both MDR and non MDR group was respiratory distress accounting for $54.84 \%$ of the total. Feed intolerance was seen in 3(9.68\%) in the MDR group and 6(19.35\%) in the non MDR group. Fever was seen in 3(9.68\%) of MDR and 2(6.45\%) of non MDR group. Lethargy was seen in $2(6.45 \%)$ of MDR and $1(3.23 \%)$ of non MDR group. There was no significant difference $(p=0.092)$ in the symptomatology in the two groups.
\end{abstract}

Keywords: MDR, neonates, antimicrobial Sensitivity

\section{Introduction}

Multidrug resistance in neonatal sepsis is a serious global issue. A group of international experts from the European Center for Disease prevention and Control (ECDC) and Center for Disease Control and prevention (CDC) in unison put an effort to give a standardized international terminology to define MDR. MDR is defined as acquired non susceptibility to at least one agent in 3 or more anti-microbial categories. Also bacteria is characterized as MDR when they are resistant to any one key antimicrobial agent, because of cross or coresistance to other agents like Methicillin Resistant Staphylococcus Aureus (MRSA) ${ }^{[1]}$.

There are very few studies done to evaluate the global burden of MDR organisms in neonatal sepsis. In one of the study done in 2010 on the long term trends in epidemiology of neonatal sepsis and antibiotic susceptibility of causative agents, the majority of gram positive isolates were found to be oxacillin resistant, but Vancomycin susceptible(MRSA) ${ }^{[2]}$.

In a study done in IPGMER Kolkata, multidrug resistance was studied in gram negative organisms in early neonatal sepsis and $>80 \%$ of early onset gram negative organisms showed in vitro resistance to ampicillin, third generation cephalosporins and gentamicin i.e. were found to be MDR. In another study done in KGMC, Lucknow in 2002 on antimicrobial resistance in organisms causing neonatal sepsis there was an overall predominance of gram negative organisms ${ }^{[3]}$. More than $89 \%$ of the Staphylococci isolated were found to be MRSA, while in Enterobatericaespp more than $40 \%$ were resistant to extended spectrum cephalosporins. Ciprofloxacin and amikacin resistance was infrequent. In a study done by Nalini et al., there was a change in the infective organisms causing early onset sepsis from gram +ve to gram -ve bacteria. Also there was an emergence of multidrug resistant Acinetobacter sp. In Safdarjung Hospital in 2011, a study done showed rise in the incidence of ESBL positive sepsis to be $37 \%{ }^{[4]}$.

There have been various studies in the direction of changing trends of antimicrobial sensitivity of organisms causing neonatal sepsis, though there have been no cumulative studies to demonstrate the risk factors, clinical profiles of neonates suffering from MDR and non MDR sepsis. Also there is no comparative data on the outcomes of MDR and non MDR neonatal sepsis. The present study aims to fill the aforementioned gaps ${ }^{[5]}$.

WHO's 2014 report on global surveillance of antimicrobial resistance reveals that antibiotic 
resistance is no longer a prediction for the future; it is happening right now, across the world, and is putting at risk the ability to treat common infections in the community and hospitals. Without urgent, coordinated action, the world is heading towards a post-antibiotic era, in which common infections and minor injuries, which have been treatable for decades, can once again kill.

Many of the bacterial pathogens associated with epidemics of human disease have evolved into multidrug-resistant (MDR) forms subsequent to antibiotic use. The term "superbugs" refers to microbes with enhanced morbidity and mortality due to multiple mutations endowing high levels of resistance to the antibiotic classes specifically recommended for their treatment; the therapeutic options for these microbes are reduced, and periods of hospital care are extended and more costly. In some cases, super resistant strains have also acquired increased virulence and enhanced transmissibility. Realistically, antibiotic resistance can be considered a virulence factor ${ }^{[6]}$.

The most prevalent Gram-negative pathogens, such as Escherichia coli, Salmonella enterica, and Klebsiellapneumoniae, cause a variety of diseases in humans and a strong correlation between antibiotic use in the treatment of these diseases and antibiotic resistance development has been observed over the past half-century. This is especially apparent with the $\beta$-lactam class of antibiotics and their related inactivating enzymes, the $\beta$ lactamases. At this time, several groups and classes have been identified, comprising up to 1,000 resistance-related $\beta$ lactamases. These include novel classes of genes and their mutant radiations.

\section{Methodology}

A prospective hospital based study over a period of one year was carried out at neonatal intensive care unit of our hospital, a tertiary care center. At least $20 \%$ deliveries require nursery admission. All neonates born at the hospital and presenting with signs and symptoms of sepsis or born to mothers with potential risk factors for sepsis were investigated for sepsis. All the neonates diagnosed to have culture positive sepsis or clinical sepsis with multidrug resistant organisms were included as study cases. For each case, the subsequent neonate with sepsis with non-MDR organisms were enrolled as control. Written informed consent was taken from parents of all cases and controls. The data for these participants was obtained by doing retrospective evaluation of medical records following a structured survey (Annexure 1) that indicated mother's age, gestational age, sex, weight at birth, risk factors for infection in newborn, clinical features, laboratory results, treatment given and outcome (discharge/death) of the baby. The diagnosis of sepsis and multidrug resistance was as per CDC criteria. Also, the risk factors which may be significantly associated with multidrug resistance like meconium stained liquor, birth asphyxia, premature rupture of membranes, fever and indiscriminate use of antibiotics in mother were studied. These newborns with sepsis were investigated and managed as per existing unit policy, guided by culture and sensitivity. Blood cultures were processed using the standard technique described by Cruickshank et al. and the antibiotic sensitivity was performed by KirbyBauer's disc diffusion method. The Parameters of morbidity included day of starting feeds after initiating antibiotics and duration of nursery stay. Outcome was taken as whether the neonate got discharged or did not survive.

Inclusion criteria: All the neonates born in Hospital and presenting with clinical signs and symptoms of sepsis were taken in this study. Also those babies born to mothers with potential risk factors were screened for sepsis were included. Each patient was included as a case patient only once if a culture positive is obtained at more than one occasions i.e. only the first episode of infection was taken into account.

\section{Results}

Among the various risk factors for neonatal sepsis, history of leaking per vaginum and fever in the mother, history of chorioamnionitis in the mother and urinary tract infection was studied as a variable. There was no significant difference noticed in the two groups with regard to risk factors for sepsis in the mother $(\mathrm{p}=0.823)$

Table 1: Showing risk factors for sepsis in the two groups

\begin{tabular}{|c|c|c|c|c|}
\hline \multirow{2}{*}{$\begin{array}{c}\text { Risk factors } \\
\text { for sepsis }\end{array}$} & \multicolumn{2}{|c|}{ Class } & \multirow{2}{*}{ Total } & \multirow{2}{*}{$\begin{array}{c}\text { P } \\
\text { value }\end{array}$} \\
\cline { 2 - 4 } No & $23(76.67 \%)$ & $23(74.19 \%)$ & $46(75.41 \%)$ & \\
Yes & $7(23.33 \%)$ & $8(25.81 \%)$ & $15(24.59 \%)$ & 0.823 \\
\cline { 2 - 4 } Total & $30(100.00 \%)$ & $31(100.00 \%)$ & $61(100.00 \%)$ & \\
\hline
\end{tabular}

Antenatal antibiotics were given to a total of 11/61(18.03\%) of mothers who had some risk factor for sepsis in newborn. However no significant increase in multidrug resistance was seen in the neonates which developed sepsis

Table 2: Showing the use of antenatal antibiotics in the two groups

\begin{tabular}{|c|c|c|c|c|c|}
\hline & \multicolumn{2}{|c|}{ Class } & \multirow{2}{*}{ Total } & \multirow{2}{*}{\begin{tabular}{|c|}
$\mathbf{P}$ \\
value
\end{tabular}} \\
\hline & & MDR & Non-MDR & & \\
\hline \multirow{2}{*}{$\begin{array}{c}\text { Antenatal } \\
\text { Antibiotics }\end{array}$} & No & $24(80.00 \%)$ & $26(83.87 \%)$ & $50(81.97 \%)$ & \multirow{3}{*}{0.694} \\
\hline & Yes & $6(20.00 \%)$ & $5(16.13 \%)$ & $11(18.03 \%)$ & \\
\hline Total & & \multicolumn{3}{|c|}{\begin{tabular}{|c|c|c|}
$30(100.00 \%)$ & $31(100.00 \%)$ & $61(100.00 \%)$ \\
\end{tabular}} & \\
\hline
\end{tabular}

Prematurity is a known risk factor for the development of sepsis in newborn. However in the present study there was no association seen with prematurity to the development of infections by a multidrug resistant organism.

Table 3: Showing the effect of prematurity on MDR and NonMDR sepsis

\begin{tabular}{|c|c|c|c|c|}
\hline & \multicolumn{2}{|c|}{ Class } & \multirow{2}{*}{ Total } & \multirow{2}{*}{ P } \\
\cline { 2 - 3 } & MDR & Non-MDR & & \\
\hline Preterm & $13(43.33 \%)$ & $13(41.94 \%)$ & $26(42.62 \%)$ & \\
\hline Term & $17(56.67 \%)$ & $18(58.06 \%)$ & $35(57.38 \%)$ & 0.912 \\
\hline Total & $30(100.00 \%)$ & $31(100.00 \%)$ & $61(100.00 \%)$ & \\
\hline
\end{tabular}

The most common presenting symptom in both MDR and non MDR group was respiratory distress accounting for $54.84 \%$ of the total. Feed intolerance was seen in 3(9.68\%) in the MDR group and 6(19.35\%) in the non MDR group. Fever was seen in $3(9.68 \%)$ of MDR and $2(6.45 \%)$ of non MDR group. Lethargy was seen in $2(6.45 \%)$ of MDR and $1(3.23 \%)$ of non MDR group. There was no significant difference $(\mathrm{p}=0.092)$ in the symptomatology in the two groups. 
Table 4: Showing the various presenting symptoms in the two classes

\begin{tabular}{|c|c|c|c|c|}
\hline \multirow{2}{*}{ Symptom } & \multicolumn{2}{|c|}{ Class } & \multirow{2}{*}{ Total } & \multirow{2}{*}{ P Value } \\
\hline & MDR & Non-MDR & & \\
\hline Abdominal Distension & $1(3.23 \%)$ & $4(12.90 \%)$ & $5(8.06 \%)$ & \multirow{8}{*}{0.092} \\
\hline Apnoea & $0(0.00 \%)$ & $2(6.45 \%)$ & $2(3.23 \%)$ & \\
\hline Feed Intolerance & $3(9.68 \%)$ & $6(19.35 \%)$ & $9(14.52 \%)$ & \\
\hline Fever & $3(9.68 \%)$ & $2(6.45 \%)$ & $5(8.06 \%)$ & \\
\hline Lethargy & $2(6.45 \%)$ & $1(3.23 \%)$ & $3(4.84 \%)$ & \\
\hline Pustules $>10$ & $0(0.00 \%)$ & $3(9.68 \%)$ & $3(4.84 \%)$ & \\
\hline Respiratory Distress & $22(70.97 \%)$ & $12(38.71 \%)$ & $34(54.84 \%)$ & \\
\hline Seizure & $0(0.00 \%)$ & $1(3.23 \%)$ & $1(1.61 \%)$ & \\
\hline
\end{tabular}

\section{Discussion}

Risk factors which are known to cause neonatal sepsis like history of leaking per vaginum> $24 \mathrm{hrs,} \mathrm{fever,} \mathrm{UTI,} \mathrm{and}$ chorioamnionitis in the mother, were studied in the 2 groups. However, there was no significant association of the above mentioned factors with multi drug resistance. The presence of meconium stained liquor and birth asphyxia during delivery were also found to be similar in the two groups. Again, low birth weight and prematurity in the neonate were not found to be significantly different in the two groups. Overall, there was no identifiable risk factor for the development of multidrug resistance in our study in the antenatal, natal and postnatal period ${ }^{[7]}$.

In our study, there was a predominance of gram positive organisms like CONS, Staph. Aureus, Micrococcus, and Enterococcus, making up to $62.29 \%$, and the gram negative organisms like E.Coli, Klebsiella and Acinetobacter amounted to $37.70 \%$. This was in contrast to the expected predominance of gram negative organisms as seen in study done by Nalini et al., (58.5\%) and S.Rehman et al. (70.4\%). However, multidrug resistance was more commonly noted in gram negative organisms (82.6\%) as compared to gram positive organisms (30.5\%). This is in coherence with the results seen in study done by Ramesh Bhat $\mathrm{Y}$ et al., in which gram negative organisms were seen to have increased to antibiotics. To the best of our knowledge, the prevalence of multidrug resistance as a whole, has not been studied earlier ${ }^{[8]}$.

In the CSF culture, $3.28 \%$ showed CONS, and $1.64 \%$ showed Staph. Aureus. However, there was no multidrug resistant organism seen in CSF culture growth.

In our study, there was a significant difference in the presence of leucopenia $(\mathrm{p}=0.006)$ and $\mathrm{I} / \mathrm{T}$ ratio $(\mathrm{p}=0.036)$ in the two groups but the levels of CRP were not significantly different $(p=0.36)$. The overall sepsis screen positivity was significantly higher in the group MDR $(p<0.005)$ this is an indicator that a positive sepsis screen should raise the suspicion of infection by an organism that is likely to be multidrug resistant.

In the CSF biochemical and cytological analysis there was no difference in the positivity rates in the two groups.

Morbidity was measured in terms of mean duration of stay in the nursery, line of antibiotic required, time of starting first feed and development of complications like DIC and thrombocytopenia.

The mean duration of stay in neonatal ICU was not found to be significantly different in two groups $(\mathrm{p}=0.276)$. But the time of starting feeds was found to be significantly earlier in the non-MDR group $(\mathrm{p}=0.025)$. This is an indicator of earlier recovery in neonates with non-MDR sepsis in whom there was a privilege of starting the oral feeds earlier. Third line antibiotics were needed for most of the cases of MDR sepsis and the difference was statistically significant. $(p=<0.005)$. There has not been any data available on these parameters pertaining to multidrug resistance in any previous studies ${ }^{[9]}$.

As expected from the available literature the mortality was found to be significantly raised in the neonates affected by MDR organisms. Similar results were seen in studies done by $\mathrm{Ni}$ Chungee $e t$ al. in which the case fatality rate was $20.7 \%$. The most common complication associated with MDR sepsis was seen to be DIC which was also the most important cause of death ${ }^{[10]}$.

\section{Conclusion}

The risk factors for neonatal sepsis during the antenatal, natal and postnatal period do not differ significantly in regard to infection by MDR organisms.

\section{References}

1. Vesikari T, Janas M, Gronroos P, Tuppurainen N, Renlund M, Kero P et al. Neonatal septicaemia. Arch Dis Child 1985;60(6):542-6.

2. Bang AT, Bang RA, Baitule SB, Reddy MH, Deshmukh MD. Effect of home-based neonatal care and management of sepsis on neonatal mortality: Field trial in rural India. Lancet 1999;354(9194):1955-61.

3. Sundaram V, Kumar P, Dutta S, Mukhopadhyay K, Ray P, Gautam V et al. Blood culture confirmed bacterial sepsis in neonates in a north Indian tertiary care center: Changes over the last decade. Jpn J Infect Dis 2009;62(1):46-50.

4. Teresa CH, Mary A, Margaret Dudeck A. CDC/NHSN surveillance definition of health care-associated infection and criteria for specific types of infections in the acute care settings. Am J Infect Control 2008;36:309-32.

5. Singh M, Narang A, Bhakoo On. Predictive Perinatal score in the diagnosis of neonatal sepsis. J Trop Pediatr 1994;40(6):365-8.

6. Aggarwal R, Sarkar N, Deorari AK, Paul VK. Sepsis in the newborn. Indian J Pediatr 2001;68(12):1143-7.

7. Kuruvilla KA, Pillai S, Jesudason M, Jana AK. Bacterial profile of sepsis in a neonatal unit in South India. Indian Pediatr 1998;35:851-858.

8. Moreno MT, Vargas S, Poveda R, Sáez-Llorens X. Neonatal sepsis and meningitis in a developing Latin American country. Pediatr Infect Dis J 1994;13(6): 516-20.

9. De Louvois, Harvey D. Infection in the new born. London: John Wiley and Sons 1990;50:107-15.

10. The Lancet Infectious Disease Commision for Antibiotic Resistance, The need for global solutions. Lancet Infect Dis 2013;13:1057-98. 